Citation: Q. Ye, W. Zhang, J. Jia, X. Li, Y. Zhou, C. Han, X. Wu, J. Yan (2021) Fungal pathogens associated with black foot of grapevine in China. Phytopathologia Mediterranea 60(2): 303-319. doi: 10.36253/phyto-12353

Accepted: April 11, 2021

Published: September 13, 2021

Copyright: () 2021 Q. Ye, W. Zhang, J. Jia, X. Li, Y. Zhou, C. Han, X. Wu, J. Yan. This is an open access, peerreviewed article published by Firenze University Press (http://www.fupress. $\mathrm{com} / \mathrm{pm}$ ) and distributed under the terms of the Creative Commons Attribution License, which permits unrestricted use, distribution, and reproduction in any medium, provided the original author and source are credited.

Data Availability Statement: All relevant data are within the paper and its Supporting Information files.

Competing Interests: The Author(s) declare(s) no conflict of interest.

Editor: Josep Armengol Forti, Polytechnical University of Valencia, Spain.

\section{Research Papers \\ Fungal pathogens associated with black foot of grapevine in China}

\author{
Qingtong YE ${ }^{1,2}$, Wei ZHANG ${ }^{1}$, Jingyi JIA ${ }^{1}$, Xinghong LI ${ }^{1}$, Yueyan \\ $\mathrm{ZHOU}^{1,2}$, ChangPing HAN ${ }^{1,2}$, XueHong WU ${ }^{2}$, JiYe YAN ${ }^{1, *}$ \\ ${ }^{1}$ Beijing Key Laboratory of Environment Friendly Management on Fruit Diseases and \\ Pests in North China, Institute of Plant and Environment Protection, Beijing Academy of \\ Agriculture and Forestry Sciences, Beijing 100097, China \\ ${ }^{2}$ Department of Plant Pathology, China Agricultural University, Beijing 100193, China \\ ${ }^{*}$ Corresponding author. E-mail: jiyeyan@vip.163.com
}

\begin{abstract}
Summary. Grapevine trunk diseases (GTDs) are the most destructive diseases in grape-growing regions worldwide. Black foot is one of the important GTDs affecting young vineyards and nurseries. This disease has not been reported in China. During 2017 and 2019, field surveys were carried out in the Guangxi, Hebei, Ningxia, Shanxi, and Xinjiang provinces of China. Incidence of plants with black foot symptoms was $0.1 \%$ to $1 \%$ in the surveyed vineyards. Plant samples with poorly developed shoots and canes, chlorotic leaves, and necrotic trunks or roots were collected from the five provinces. In total, 50 fungal isolates were obtained from symptomatic tissues. Based on morphological and multi-gene phylogenetic analyses, five species were identified as Cylindrocladiella lageniformis, Dactylonectria torresensis, D. macrodidyma, D. alcacerensis and Neonectria sp.1. Pathogenicity was assessed using young, healthy detached green shoots of grapevine 'Summer Black' and potted 3-month-old 'Summer Black' cuttings. Inoculated detached shoots developed necroses after $7 \mathrm{~d}$, and inoculated cuttings after $80 \mathrm{~d}$. Fungi were re-isolated from necrotic lesions. Among the five species, D. macrodidyma was the most aggressive. This is the first report of C. lageniformis, D. torresensis, D. macrodidyma, D. alcacerensis, and Neonectria sp. 1 associated with black foot in China. This study has enhanced knowledge of the fungi associated with black foot in China, and will assist development of control measures for this disease.
\end{abstract}

Keywords. Nectriaceae, morphological characteristics, phylogenetic analyses, Vitis vinifera.

\section{INTRODUCTION}

Grapevine (Vitis vinifera L.) is an economically important fruit crop, with global cultivation area of 7,449,000 hectares in 2018, and China is ranked the second in the world grapevine cultivation area (2019 OIV). More than 70 diseases have been reported in grapevines, most of which are caused by fungi or oomycetes (Wilcox et al., 2006), and among these, at least 27 diseases have been reported in China. Esca complex, Botryosphaeria dieback, black foot (BF), Eutypa dieback, and Phomopsis dieback are major fungal 
grapevine trunk diseases (GTDs) worldwide. These diseases have been reported in almost all the main grapegrowing countries (Gramaje et al., 2018). GTDs are complexes that affect grape yields, wine quality and lifespan of plants in many grape-growing regions. The global financial losses attributed to GTDs are estimated to be more than \$US 1.5 billion per year (Hofstetter et al., 2012).

Black foot $(\mathrm{BF})$ is one of the most significant GTDs, especially in nurseries and young plantations (Halleen et al., 2006). This disease has occurred in many viticulture regions during the last decade, including Australia, Brazil, California, Canada, (British Columbia, Quebec), France, Iran, Italy, New Zealand, Portugal, South Africa (Western Cape), Spain, Switzerland, Turkey, United States of America, and Uruguay (Agustí-Brisach and Armengol, 2013; Lombard et al., 2014; Carlucci et al., 2017; Aigoun-Mouhous et al., 2019; Lawrence et al., 2019; Berlanas et al., 2020). In France, 50\% of losses caused by BF fungal pathogens were recorded in young vineyards of 2 to 8 years old (Larignon et al., 1999). In the Czech Republic, about 30\% of plants showed root necroses, reduced root biomass and wood necroses in the basal ends of grapevine rootstocks, which are typical symptoms of BF that appear after one year of cultivation in 2015 (Pecenka et al., 2018). The most common symptoms of BF on grapevine plants include delayed or absent budding, stunted growth, shortened internodes with small trunks, chlorotic leaves with necrotic margins, brown to black necroses on rootstock bases and sunken necrotic root lesions, in nurseries and young plantations (Rego et al., 2000; Halleen et al., 2006; Alaniz et al., 2007; Abreo et al., 2010; Agustí-Brisach and Armengol, 2013).

Ilyonectria destructans (=Cylindrocarpon destructans) was first reported in France (Grasso and Magnano Di San Lio, 1975), and to date, 33 fungal species have been reported to be associated with BF. The most common fungal genera associated with $\mathrm{BF}$ are Campylocarpon, Cylindrocladiella, Dactylonectria, Ilyonectria, Neonectria, Pleiocarpon and Thelonectria. Fungal species that have been associated with BF include: Campylocarpon fasciculare, Ca. pseudofasciculare, "Cylindrocarpon" sp. 2, Cylindrocarpon didymium, Cylindrocladiella parva, C. lageniformis, C. viticola, and C. peruviana, Dactylonectria alcacerensis, D. estremocensis, D. macrodidyma, D. novozelandica, D. pauciseptata, D. pinicola, $D$. torresensis, $D$. riojana, $D$. vitis, $D$. hordeicola, Ilyonectria destructans, I. europaea, I. liriodendri, I. lusitanica, I. pseudodestructans, I. robusta, I. vivaria, Neonectria obtusispora, $N$. quercicola, Neonectria sp. 1, fungi in the N. mammoidea group, Pleiocarpon algeriense.
Thelonectria blackeriella, T. olida, and T. aurea. (AgustíBrisach and Armengol, 2013; Lombard et al., 2014; Carlucci et al., 2017; Aigoun-Mouhous et al., 2019; Lawrence et al., 2019; Berlanas et al., 2020). Among these species, I. liriodendri and D. macrodidyma are the most widely distributed ones (Agustí-Brisach and Armengol, 2013). These fungi are frequently isolated from BF symptoms in nursery and older grapevine plants (Petit et al., 2011; Carlucci et al., 2017), and from asymptomatic inner tissues from plants (Berlanas et al., 2020). Some BF fungi have also been detected from the soils of grapevine nurseries and vineyards, in Spain and South Africa. (AgustíBrisach et al., 2013, 2014; Langenhoven et al., 2018).

Eutypa dieback was first reported in China in 2007, Botryosphaeria dieback in 2010, Diaporthe dieback in 2015 and Esca in 2020 (Li et al., 2007; Li et al., 2010; Dissanayake et al., 2015; Ye et al., 2020), while BF has not been reported in China previously.

\section{MATERIALS AND METHODS}

\section{Vineyard surveys}

Surveys were carried out in ten vineyards, located in Ningxia, Hebei, Shanxi, Guangxi and Xinjiang provinces of China, during 2017 and 2019 (Figure 1a). These provinces belong to the top ten grape cultivated grapevine areas in China, and Xinjiang province ranked the first, followed by Hebei province. The training systems used in the surveyed vineyards was mini "J". The vineyards were of similar age, from 5 to 6 years old. Typical symptoms associated with diseased vines were shortened shoot internodes, chlorotic leaves, and trunk and root necroses (Figure 1, b-h). Initial disease symptoms included root necroses (especially small roots). As the disease progressed, the above-ground plant parts developed shoot shortened internodes and chlorotic leaves in severe cases. Some grapevines were grafted (rootstock Fercal), and some others were self-rooted (Personal communication, some of the grape growers).

Sample collection, fungus isolation and morphology of the pathogens

Samples were collected from V. rotundifolia Michx., and V. vinifera cvs Marselan, Cabernet Franc or Cabernet Sauvignon. Typical symptoms were recorded by taking appropriate photographs. The samples were kept at $4^{\circ} \mathrm{C}$ for further study, and the presence of spores or structures on the surfaces of trunks or roots were detected using a microscope. Isolations were made from 


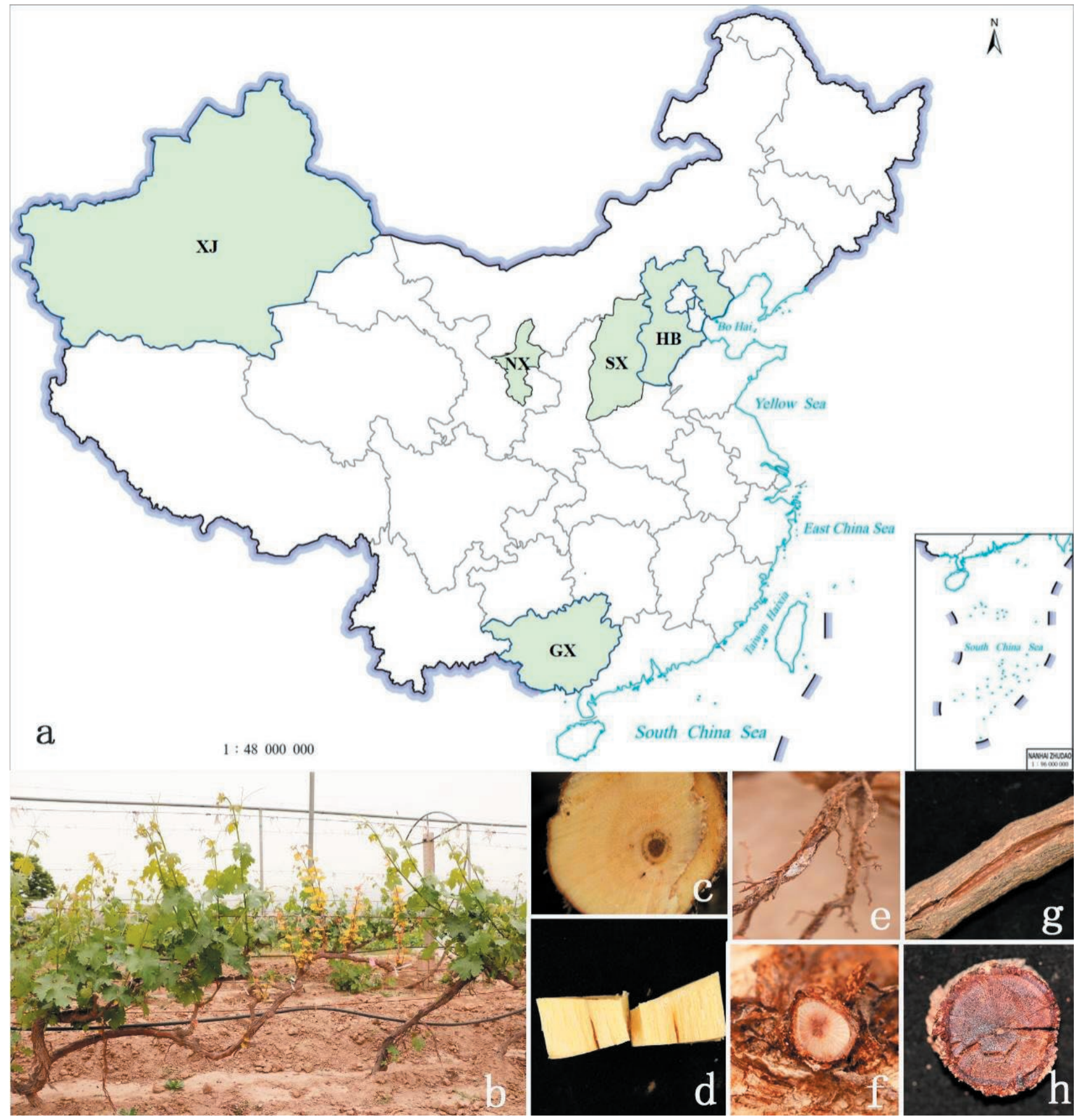

Figure 1. Sample collection sites and disease symptoms. Field surveys were carried out in five provinces of China. GX: Guangxi; HB: Hebei; NX: Ningxia;SX: Shanxi; XJ: Xinjiang (a). Diseased plant (Chardonnay, 5-year-old) showing shortened internodes of shoots (b).Necrotic grapevine roots and trunks $(\mathbf{c}-\mathbf{h})$.

symptomatic trunks and roots. Necrotic root and trunk samples were debarked and cut into small pieces (4-5 $\mathrm{mm}^{2}$ ). These small pieces were then surface-sterilized in $75 \%$ ethanol for $30 \mathrm{~s}$, rinsed three times with sterilized water, dried, and cultured on potato dextrose agar (PDA; $20 \%$ potatoes, $2 \%$ dextrose, 1.5 to $2 \%$ agar) in Petri plates. The plates were incubated at $25^{\circ} \mathrm{C}$. Fungi growing from tissue pieces were transferred onto new PDA plates after $7 \mathrm{~d}$, and pure cultures were obtained by isolating single spores. Pure cultures were grown on PDA and malt extract agar (MEA) and incubated at $25^{\circ} \mathrm{C}$ in the dark for $7 \mathrm{~d}$. Conidia and colonies on the MEA plates 
were observed and photographed using the Axio Imager Z2 photographic microscope (Carl Zeiss Microscopy).

\section{DNA amplification and phylogenetic analyses}

Single-spore purification were done for all the isolates before DNA extractions. Total genomic DNA was extracted from 50-100 mg of mycelium after $14 \mathrm{~d}$ of incubation on PDA (Guo et al., 2000). For initial genus identification, the internal transcribed spacer and intervening $5.8 \mathrm{~S}$ gene regions (ITS) were amplified and sequenced for all the isolates, and the resulting sequences were searched using BLASTN within GenBank/NCBI (https://blast.ncbi.nlm.nih.gov/Blast.cgi), as described by Manawasinghe et al. (2019). All the isolates in the present study belonged to Cylindrocladiella, Neonectria or Dactylonectria.

For species confirmation, phylogenetic analyses were conducted using multigene phylogenies. For Cylindrocladiella, histone $\mathrm{H} 3$ (his3), $\beta$-tubulin (tub2), and partial translation elongation factor 1-alpha (tef1) were sequenced (Marin-Felix et al., 2019). ITS, tub2, his 3 , and tef1 gene regions were sequenced for Dactylonectria and Neonectria species (Berlanas et al., 2020). The primer pairs and amplification protocols used in the present study are summarized in Table 1. Each PCR mixture comprised $1.0 \mu \mathrm{L}$ of genomic DNA, 0.6 $\mu \mathrm{L}$ of TaKaRa ExTaq DNA polymerase, $5.0 \mu \mathrm{L}$ of $10 \times$ ExTaq DNA polymerase buffer, $4.0 \mu \mathrm{L}$ of dNTPs, and $1.0 \mu \mathrm{L}$ of each primer, and was adjusted with sterilized double-distilled water to a final volume of $50.0 \mu \mathrm{L}$. The PCR reactions were carried out in a thermal cycler (Bio-Rad, model C1000). Amplification products were visualized on $1 \%$ agarose electrophoresis gels under UV light using a Gel DocTM XR+ Molecular Imager (BioRad). All positive bands obtained by PCR amplification were sequenced by Tsingke Company, Beijing, China, and the sequence data obtained were deposited in GenBank (Table 2).

Reference sequences of related taxa were obtained from GenBank (Marin-Felix et al., 2019; Berlanas et al., 2020). The sequence data generated in the present study were included, and individual gene regions were aligned using the MAFFT v. 7 webserver (Kuraku et al., 2013; Katoh et al., 2019) (https://mafft.cbrc. jp/alignment/server/). The alignments were checked and edited manually, where necessary using BioEdit v7.0.9 (Hall, 1999). Phylogenetic trees were generated using Maximum Likelihood (ML) in RAxML (Silvestro and Michalak, 2016) and Maximum Parsimony (MP) in PAUP (v4.0) (Swofford, 2002). The ML and MP trees were constructed using the methods described by Manawasinghe et al. (2019). For MP, heuristic searches were conducted with 1000 bootstrap replicates by random addition. All characters were unordered and equally weighted. Gaps were treated as missing data, and the steepest descent option not in effect, whereas the MulTrees option was used. The Tree Length (TL), Consistency Index (CI), Retention Index (RI), Relative Consistency Index (RC), and Homoplasy Index (HI) were calculated in PAUP. All the resulting trees were saved and checked using Kishino-Hasegawa tests (Kishino and Hasegawa, 1989). The ML analyses of single genes and combined multiple genes were accomplished using the RAxML-HPC2 on XSEDE (8.2.8) in the CIPRES Science Gateway (https://www.phylo.org/ portal2/createTask!create.action). Phylogenetic trees were visualized using FigTree v1.4.4 (Rambaut, 2018) and were annotated in Microsoft PowerPoint 2016.

\section{Pathogenicity tests}

Pathogenicity tests of potential BF pathogens were conducted on detached green shoots or potted 3-month-

Table 1. The primer pairs and their amplified protocols used in present study.

\begin{tabular}{|c|c|c|c|c|}
\hline Gene region & Primers & Sequence 5'-3' & Protocols for PCR & References \\
\hline \multirow[t]{2}{*}{ ITS } & ITS1 & TCCGTAGGTGAACCTGCGG & \multirow{2}{*}{$\begin{array}{l}94^{\circ} \mathrm{C}: 3 \mathrm{~min},\left(94^{\circ} \mathrm{C}: 30 \mathrm{~s}, 52^{\circ} \mathrm{C}: 30 \mathrm{~s}\right. \\
\left.72^{\circ} \mathrm{C}: 1 \mathrm{~min}\right) \times 34 \text { cycles } 72^{\circ} \mathrm{C}: 7 \mathrm{~min}\end{array}$} & \multirow[t]{2}{*}{ White et al. (1990) } \\
\hline & ITS4 & TCCTCCGCTTATTGATATGC & & \\
\hline \multirow[t]{2}{*}{ HIS } & CYLH3F & AGGTCC ACTGGTGGCAAG & \multirow{2}{*}{$\begin{array}{l}94^{\circ} \mathrm{C}: 3 \mathrm{~min},\left(94^{\circ} \mathrm{C}: 30 \mathrm{~s}, 58^{\circ} \mathrm{C}: 30 \mathrm{~s}\right. \\
\left.72^{\circ} \mathrm{C}: 1 \mathrm{~min}\right) \times 34 \text { cycles } 72^{\circ} \mathrm{C}: 7 \mathrm{~min}\end{array}$} & \multirow[t]{2}{*}{ Crous et al. (2004) } \\
\hline & CYLH3R & AGCTGGATGTCCTTGGACTG & & \\
\hline \multirow[t]{2}{*}{$\beta$-tubulin } & T1 & AACATGCGTGAGATTGTAAGT & \multirow{2}{*}{\multicolumn{2}{|c|}{$\begin{array}{l}94^{\circ} \mathrm{C}: 3 \mathrm{~min},\left(94^{\circ} \mathrm{C}: 30 \mathrm{~s}, 58^{\circ} \mathrm{C}\left(62^{\circ} \mathrm{C}\right): 30 \mathrm{O}^{\prime} \text { Donnell and Cigelnik (1997) }\right. \\
\left.\mathrm{s}, 72^{\circ} \mathrm{C}: 1 \mathrm{~min}\right) \times 34 \text { cycles } 72^{\circ} \mathrm{C}: 7 \mathrm{~min} \quad \text { Glass and Donaldson }(1995)\end{array}$}} \\
\hline & Bt $2 b$ & ACCCTCAGTGTAGTGACCCTTGGC & & \\
\hline \multirow[t]{2}{*}{ EF1- $a$} & EF1-728F & CATCGAGAAGTTCGAGAAGG & \multirow{2}{*}{$\begin{array}{l}94^{\circ} \mathrm{C}: 3 \mathrm{~min},\left(94^{\circ} \mathrm{C}: 30 \mathrm{~s}, 54^{\circ} \mathrm{C}: 30 \mathrm{~s}\right. \\
\left.72^{\circ} \mathrm{C}: 1 \mathrm{~min}\right) \times 34 \text { cycles } 72^{\circ} \mathrm{C}: 7 \mathrm{~min}\end{array}$} & Carbone and Kohn (1999) \\
\hline & EF1-986R & TACTTGAAGGAACCCTTACC & & Udayanga et al. (2012a ; b) \\
\hline
\end{tabular}


Table 2. Reference sequence data obtained from GenBank and isolate sequence data from the present study which were used for phylogenetic tests.

\begin{tabular}{|c|c|c|c|c|c|}
\hline \multirow{2}{*}{ Species } & \multirow{2}{*}{ Isolates } & \multicolumn{4}{|c|}{ GenBank accession No. } \\
\hline & & ITS & tub2 & his3 & tef1 \\
\hline Neonectria coccinea & CBS 119158 & JF268759 & KC660727 & $\mathrm{N} / \mathrm{A}$ & DQ789749.1 \\
\hline N. confusa & CBS 127484 & KM515889 & KM515886 & N/A & N/A \\
\hline N. confusa & CBS 127485 & FJ560437 & FJ860054 & $\mathrm{N} / \mathrm{A}$ & JF268736.1 \\
\hline N. ditissima & CBS 226.31 & JF735309 & DQ789869 & JF735594 & JF735783 \\
\hline N. ditissima & CBS 835.97 & JF735310 & DQ789880 & JF735595 & JF735784 \\
\hline N. faginata & CBS 217.67 & HQ840385 & JF268730 & N/A & JF268746.1 \\
\hline N. faginata & CBS 119160 & HQ840384 & DQ789883 & N/A & N/A \\
\hline N. fuckeliana & CBS 119200 & HQ840387 & JF268731 & N/A & JF268747.1 \\
\hline N. fuckeliana & CBS 239.29 & HQ840386 & DQ789871 & N/A & JF268748.1 \\
\hline N. hederae & CBS 714.97 & N/A & DQ789878 & N/A & KC660461 \\
\hline N. hederae & IMI 058770 & N/A & DQ789895 & N/A & DQ789752 \\
\hline N. lugdunensis & CBS 125475 & KM231762 & KM232019 & KM231482.1 & KM231887.1 \\
\hline N. lugdunensis & CBS 125485 & KM231762 & KM232019 & KM231482 & KM231887 \\
\hline N. major & CBS 240.29 & JF735308 & DQ789872 & JF735593 & JF735782 \\
\hline N. neomacrospora & CBS 198.62 & AJ009255 & HM352865 & KM231481 & HM364351 \\
\hline N. neomacrospora & CBS 324.61 & JF735312 & DQ789875 & JF735599 & JF735788 \\
\hline N. neomacrospora & CBS 503.67 & AY677261 & JF735436 & JF735600 & JF735789 \\
\hline N. obtusispora & CBS 183.36 & AM419061 & AM419085 & JF735607 & JF735796 \\
\hline N. obtusispora & СРС 13544 & AY295306 & JF735443 & JF735608 & JF735797 \\
\hline N. punicea & CBS 242.29 & KC660522 & DQ789873 & N/A & DQ789730 \\
\hline N. punicea & CBS 119724 & КС660496 & DQ789824 & N/A & DQ789681 \\
\hline N. quercicola & CBS 143704 & KY676880 & KY676874 & KY676862 & KY676868 \\
\hline N. quercicola & СРС 13530 & AY295302 & JF735441 & JF735605 & JF735794 \\
\hline N. ramulariae & MAFF411012 & JX034565.1 & JX034567.1 & N/A & N/A \\
\hline N. ramulariae & CBS 151.29 & AY677291 & JF735438 & JF735602 & DQ789720 \\
\hline N. ramulariae & CBS 182.36 & HM054157 & JF735439 & JF735603 & JF735792 \\
\hline Neonectria sp. 1 & СРС 13545 & N/A & JF735437 & JF735601 & JF735790 \\
\hline Neonectria sp. 1 & JZB3210004 & MN988722 & MN958534 & MN958545 & MN956387 \\
\hline N. tsugae & CBS 788.69 & KM231763 & KM232020 & N/A & DQ789720 \\
\hline Dactylonectria alcacerensis & CBS 129087 & JF735333 & N/A & JF735630 & JF735819 \\
\hline D. alcacerensis & Cy134 & JF735332 & N/A & JF735629 & JF735818 \\
\hline D. alcacerensis & JZB3310007 & MN988716 & MN958528 & MN958539 & MN956381 \\
\hline D. amazonica & MUCL 55430 & MF683706 & MF683643 & MF683686 & MF683664 \\
\hline D. anthuriicola & CBS 564.95 & JF735302 & JF735430 & JF735579 & JF735768.1 \\
\hline D. ecuadoriensis & MUCL 55424 & MF683704 & MF683641 & MF683684 & MF683662 \\
\hline D. ecuadoriensis & MUCL55425 & MF683705 & MF683642 & MF683684 & MF683663 \\
\hline D. estremocencis & СРC 13539 & JF735330 & JF735458 & JF735627 & JF735816 \\
\hline D. estremocencis & CBS 129085 & JF735320 & JF735448 & JF735617 & JF735806 \\
\hline D. hispanica & CBS 142827 & KY676882 & KY676876 & KY676864 & KY676870 \\
\hline D. hispanica & Cy228 & JF735301 & JF735429 & JF735578 & JF735767 \\
\hline D. hordeicola & CBS 162.89 & AM419060 & AM419084 & JF735610 & JF735799 \\
\hline D. macrodidyma & CBS 112601 & MH862898 & AY677229 & JF735644 & JF735833 \\
\hline D. macrodidyma & CBS 112615 & AY677290 & AY677233 & JF735647 & JF268750 \\
\hline D. macrodidyma & Cy258 & JF735348 & JF735477 & JF735656 & JF735845 \\
\hline D. macrodidyma & CBS 112604 & AY677284 & AY677229 & JF735644 & JF735833 \\
\hline D. macrodidyma & JZB3310008 & MN988717 & MN958529 & MN958540 & MN956382 \\
\hline D. macrodidyma & JZB3310009 & MN988718 & MN958530 & MN958541 & MN956383 \\
\hline
\end{tabular}


Table 2. (Continued).

\begin{tabular}{|c|c|c|c|c|c|}
\hline \multirow{2}{*}{ Species } & \multirow{2}{*}{ Isolates } & \multicolumn{4}{|c|}{ GenBank accession No. } \\
\hline & & ITS & tub2 & his3 & tef1 \\
\hline D. macrodidyma & JZB33100010 & MN988719 & MN958531 & MN958542 & MN956384 \\
\hline D. novozelandica & CBS 112608 & AY677288 & AY677235 & JF735632 & JF735821 \\
\hline D. palmicola & MUCL55426 & MF683708.1 & MF683645.1 & MF683687.1 & MF683666.1 \\
\hline D. pauciseptata & CBS 120171 & EF607089 & EF607066 & JF735587 & JF735776 \\
\hline D. pinicola & CBS 159.43 & JF735318 & JF735446 & JF735613 & JF735802 \\
\hline D. pinicola & CBS 173.37 & JF735319 & JF735447 & JF735614 & JF735803 \\
\hline D. polyphaga & MUCL55209 & MF683689 & MF683626 & MF683668 & MF683647 \\
\hline D. torresensis & CBS 119.41 & JF735349 & JF735478 & JF735657 & JF735846 \\
\hline D. torresensis & Cyl102 & KP823905 & KP823885 & KP823894 & KP823874 \\
\hline D. torresensis & Cyl106 & KP823907 & KP823887 & KP823895 & KP823876 \\
\hline D. torresensis & Cyl110 & KP823908 & KP823888 & KP823896 & KP823877 \\
\hline D. torresensis & Cyl124 & KP823912 & KP823891 & KP823900 & KP823881 \\
\hline D. torresensis & CBS 129086 & JF735362 & JF735492 & JF735681 & JF735870.1 \\
\hline D. torresensis & JZB33100011 & MN988720 & MN958532 & MN958543 & MN956385 \\
\hline D. torresensis & JZB33100012 & MN988721 & MN958533 & MN958544 & MN956386 \\
\hline D. vitis & CBS 129082 & JF735303 & JF735431 & JF735580 & JF735769.1 \\
\hline D. valentina & CBS 142826 & KY676881 & KY676875 & KY676863 & KY676869 \\
\hline Cylindrocladiella addiensis & CBS 143794 & MH111383 & MH111388 & $\mathrm{N} / \mathrm{A}$ & MH111393 \\
\hline C. addiensis & CBS 143793 & MH111385 & MH111390 & N/A & MH111395 \\
\hline C. addiensis & CBS 143795 & MH111384 & MH111389 & $\mathrm{N} / \mathrm{A}$ & MH111394 \\
\hline C. arbusta & CMW47295/ CBS 143546 & MH017015 & MH016958 & MH016996 & MH016977 \\
\hline C. arbusta & CMW 47296; CBS 143547 & MH017016 & MH016959 & MH016997 & MH016978 \\
\hline C. australiensis & CBS 129567 & JN100624 & JN098747 & JN098932 & JN099060 \\
\hline C. brevistipitata & CBS 142786 & N/A & MF444926 & N/A & MF444940 \\
\hline C. camelliae & IMI 346845 & AF220952 & AY793471 & AY793509 & JN099087 \\
\hline C. clavata & CBS 129564 & JN099095 & JN098752 & JN098858 & JN098974 \\
\hline C. cymbiformis & CBS 129553 & JN099103 & JN098753 & JN098866 & JN098988 \\
\hline C. elegans & CBS 338.92 & AY793444 & AY793474 & AY793512 & JN099039 \\
\hline C. ellipsoidea & CBS 129573 & JN099094 & JN098757 & JN098857 & JN098973 \\
\hline C. hahajimaensis & MAFF238172 & JN687561 & N/A & N/A & JX024570.1 \\
\hline C. hawaiiensis & CBS 129569 & JN100621 & JN098761 & JN098929 & JN099057 \\
\hline C. horticola & CBS 142784 & MF444911 & MF444924 & N/A & MF444938 \\
\hline C. humicola & CBS 142779 & MF444906 & MF444919 & N/A & MF444933 \\
\hline C. infestans & CBS 111795 & AF220955 & AF320190 & AY793513 & JN099037 \\
\hline C. kurandica & CBS 129577 & JN100646 & JN098765 & JN098953 & JN099083 \\
\hline C. lageniformis & CBS 111060 & JN100611 & JN098770 & JN098918 & JN099046 \\
\hline C. lageniformis & CBS 111061 & JN100606 & JN098771 & JN098913 & JN099040 \\
\hline C. lageniformis & CBS 112898 & AY793445 & AY725652 & AY725699 & JN098990 \\
\hline C. lageniformis & CBS 340.92 & MH862360 & AY793481 & AY793520 & JN099003 \\
\hline C. lageniformis & JZB3320001 & MN988714 & MN958526 & MN958537 & MN958535 \\
\hline C. lageniformis & JZB3320002 & MN988715 & MN958527 & MN958538 & MN958536 \\
\hline C. lanceolata & CBS 129566 & JN099099 & JN098789 & JN098862 & JN098978 \\
\hline C. lateralis & CBS 142788 & MF444914 & MF444928 & N/A & MF444942 \\
\hline C. longiphialidica & CBS 129557 & JN100585 & JN098790 & JN098851 & JN098966 \\
\hline C. longistipitata & CBS 116075 & AF220958 & AY793506 & AY793546 & JN098993 \\
\hline C. malesiana & CBS 143549 & MH017017 & MH016960 & MH016998 & MH016979 \\
\hline C. microcylindrica & CBS 111794 & AY793452 & AY793483 & AY793523 & JN099041 \\
\hline C. natalensis & CBS 114943 & JN100588 & JN098794 & JN098895 & JN099016 \\
\hline
\end{tabular}


Table 2. (Continued).

\begin{tabular}{|c|c|c|c|c|c|}
\hline \multirow{2}{*}{ Species } & \multirow{2}{*}{ Isolates } & \multicolumn{4}{|c|}{ GenBank accession No. } \\
\hline & & ITS & tub2 & his3 & tef1 \\
\hline C. nederlandica & CBS 152.91 & JN100603 & JN098800 & JN098910 & JN099033 \\
\hline C. novazelandica & CBS 486.77 & AF220963 & AY793485 & AY793525 & JN099050 \\
\hline C. nauliensis & CBS 143792 & MH111387 & MH111392 & N/A & MH111397 \\
\hline C. nauliensis & CBS 143791 & MH111386 & MH111391 & $\mathrm{N} / \mathrm{A}$ & MH111396 \\
\hline C. obpyriformis & CMW47194/ CBS 143552 & MH017022 & MH016965 & MH017003 & MH016984 \\
\hline C. parvispora & CMW 47197/ CBS 143554 & MH017025 & MH016968 & MH017006 & MH016987 \\
\hline C. parva & CBS 114524 & AF220964 & AY793486 & AY793526 & JN099009 \\
\hline C. peruviana & IMUR 1843 & AF220966 & AY793500 & AY793540 & JN098968 \\
\hline C. pseudocamelliae & CBS 129555 & JN100577 & JN098814 & JN098843 & JN098958 \\
\hline C. pseudohawaiiensis & CBS 210.94 & JN099128 & JN098819 & JN098890 & JN099012 \\
\hline C. pseudoinfestans & CBS 114531 & AF220957 & AY793508 & AY793548 & JN099004 \\
\hline C. pseudoparva & CBS129560 & JN100620 & JN098824 & JN098927 & JN099056 \\
\hline C. queenslandica & CBS 129574 & JN099098 & JN098826 & JN098861 & JN098977 \\
\hline C. reginae & CBS 142782 & MF444909 & MF444922 & N/A & MF444936 \\
\hline C. solicola & $\begin{array}{l}\text { CMW47198/ } \\
\text { CBS } 143551\end{array}$ & MH017021 & MH016964 & MH017002 & MH016983 \\
\hline C. stellenboschensis & CBS 110668 & JN100615 & JN098829 & JN098922 & JN099051 \\
\hline C. terrestris & CBS 142789 & MF444915 & MF444929 & N/A & MF444943 \\
\hline C. thailandica & CBS 129571 & JN100582 & JN098834 & JN098848 & JN098963 \\
\hline C. variabilis & CBS 129561 & JN100643 & JN098719 & JN098950 & JN099080 \\
\hline C. viticola & CBS 112897 & AY793468 & AY793504 & AY793544 & JN099064 \\
\hline C. vitis & CBS 142517 & KY979751 & KY979918 & N/A & KY979891 \\
\hline Campylocarpon fasciculare & CBS 112613 & AY677301.1 & AY677221.1 & JF735502.1 & JF735691.1 \\
\hline C. pseudofasciculare & CBS 112679 & AY677306.1 & KJ022328.1 & JF735503.1 & JF735692.1 \\
\hline Gliocladiopsis sagariensis & CBS 199.55 & NR147628 & JQ666141 & JQ666031 & JQ666107 \\
\hline
\end{tabular}

a CBS: Westerdijk Fungal Biodiversity Institute, Utrecht, The Netherlands; CPC: Culture collection of Pedro Crous, housed at CBS. MUCL: Mycothèque de l'Université catholique de Louvain; JZB: Beijing Academy of Agriculture and Forestry Sciences Culture Collection, China; IMI: International Mycological Institute, CABI-Bioscience, Egham, Bakeham Lane, U.K; IMUR: Institute of Mycology, University of Recife, Recife, Brazil; MAFF: Genetic Resources Centre, National Agriculture and Food Research Organization (NARO), NARO GenBank, Ibaraki, Japan; Cy: Cylindrocarpon collection housed at Laboratório de Patologia Vegetal "Veríssimo de Almeida" - ISA, Lisbon, Portugal;

${ }^{\mathrm{b}}$ Ex-type were in bold.

N/A: The sequence is not available or not applicable to the present study.

old healthy plants of grapevine cv. 'Summer Black'. Five isolates (JZB3320001, JZB3310007, JZB3310008, JZB33100011 and JZB3210004) were selected randomly for pathogenicity tests. Mycelium discs (4 mm diam.) were obtained from the edges of PDA colonies which were grown for $10 \mathrm{~d}$ at $25^{\circ} \mathrm{C}$.

Detached shoots were surface-disinfected in $75 \%$ ethanol and then dried, and each shoot was then wounded $(4 \mathrm{~mm})$ using a sterilized scalpel. The mycelium discs were placed onto the wound sites and covered with parafilm (Bemis). Non-colonized sterile PDA plugs were used as negative controls. The shoots were then inserted into moist soil and kept at $25^{\circ} \mathrm{C}$. Each experiment included ten shoots for each fungus isolate, with a total of three parallel experiments conducted.
The lengths of the lesions were measured after $7 \mathrm{~d}$, and meanwhile photos were taken.

Pathogenicity tests of BF fungal agents were further conducted on the 3-month old grapevine cuttings which were inoculated in a manner similar to the detached green shoots. The experiment was performed in six cuttings for each tested isolate and the negative controls. The plants were kept in a greenhouse maintained at $25^{\circ} \mathrm{C}$, and the trial was conducted twice. Shoots were collected, and lesion lengths were measured upward and downward from the points of inoculation after $80 \mathrm{~d}$.

Fungi were re-isolated from necroses on the test plants in all pathogenicity tests, and fungus identifications were based on cultural and morphological characters. The lesion dimension data were statistically ana- 
lyzed with IBM SPSS Statistics 21.0 (IBM Corp.) using a one-way analysis of variance (at $P=0.05$ ) to determine differences in shoot lesion dimensions resulting from different fungus isolate inoculations.

\section{RESULTS}

Fungus isolation and initial species identifications

Incidence of BF-like symptoms in the investigated vineyards was $0.1 \%$ to $1 \%$.

Colony morphology of all the isolates distinguished after $14 \mathrm{~d}$ of growth on PDA. In total, 50 isolates were obtained from the symptomatic grapevine tissues. For genus confirmation of the isolates, the ITS regions were amplified for all the isolates. The products of the ITS regions were approx. $0.5 \mathrm{~kb}$. All sequences obtained were compared to those deposited in GenBank, and the isolates possessed $95 \%-99 \%$ similarity with sequences from the genera Cylindrocladiella, Dactylonectria or Neonectria. One or two isolates were selected from each of these three genera for pathogenicity tests.

The ML MP trees had similar topologies, so only the ML tree is presented in this study, with ML and MP bootstrap support values.

Identification of Cylindrocladiella species The optimization likelihood value of the final ML tree was -9044.171598. The matrix had 563 distinct alignment patterns, with $12.52 \%$ of undetermined characters or gaps. The parameters for the GAMMA+P-Invar model were as follows: estimated base frequencies $\mathrm{A}$ $=0.217646, \mathrm{C}=0.325293, \mathrm{G}=0.206497, \mathrm{~T}=0.250564$; substitution rates include $\mathrm{TL}=1.662695, \mathrm{AC}=1.021632$, $\mathrm{AG}=3.427269, \mathrm{AT}=1.399071, \mathrm{CG}=0.562601, \mathrm{CT}=$ $4.088874, \mathrm{GT}=1.000000$; proportion of invariable sites (I) $=0.346767$, and gamma distribution shape parameter $(\alpha)=0.401996$. In the MP tree, the heuristic search produced 1000 trees (length $=1415, \mathrm{CI}=0.529, \mathrm{RI}=0.840$, $\mathrm{RC}=0.445$ and $\mathrm{HI}=0.471$ ), and the dataset consisted of 1527 total characters. Of these characters, 1000 were constant, 158 variable characters were parsimony-uninformative and 369 were parsimony-informative. In the ML tree (Figure 2), generated using the combined data, two isolates (JZB3320001 and JZB3320002) clustered with C. lageniformis (CBS 340.92) with bootstrap value 100 and 99 obtained from the ML and MP tree, respectively. Based on morphological characteristics and phylogenetic results, isolates JZB3320001 and JZB3320002 were identified as Cylindrocladiella lageniformis Crous, M.J. Wingf. \& Alfenas.

Identification of the species of Dactylonectria and Neonectria species The ML optimization likelihood value was
-12848.031218. The matrix had 916 distinct alignment patterns, with $16.70 \%$ of undetermined characters or gaps. Parameters for the GAMMA+P-Invar model were: estimated base frequencies $\mathrm{A}=0.216108, \mathrm{C}=0.328348, \mathrm{G}=$ $0.225791, \mathrm{~T}=0.229753$; substitution rates include $\mathrm{TL}=$ 1.593283, $\mathrm{AC}=1.370476, \mathrm{AG}=3.210224, \mathrm{AT}=1.626079$, $\mathrm{CG}=0.702088, \mathrm{CT}=5.644898, \mathrm{GT}=1.000000$; proportion of invariable sites $(\mathrm{I})=0.388010$, and gamma distribution shape parameter $(\alpha)=0.971160$. In the MP tree, the heuristic search produced 1000 trees (length $=1981$, CI $=0.610, \mathrm{RI}=0.911, \mathrm{RC}=0.556$ and $\mathrm{HI}=0.390$ ), and the dataset consisted of 2041 total characters. Of these, 1225 were constant, 88 variable characters were parsimonyuninformative and 728 were parsimony-informative. In the ML tree (Figure 3), generated using the combined data, three isolates (JZB3310008, JZB3310009 and JZB33100010) collected from Ningxia province clustered with $D$. macrodidyma (CBS 112615) and one isolate (JZB3210004) clustered with Neonectria sp. 1(CPC 13545); In addition, the isolate (JZB3310007) clustered with D. alcacerensis (CBS 129087) and two isolates (JZB33100011 and JZB33100012) clustered with $D$. torresensis(CBS 119.41).

\section{Morphological characteristics}

Morphological observations for the five identified species are outlined below.

Cylindrocladiella lageniformis Crous, M.J. Wingf. \& Alfenas

Pathogenic on trunks and rootstocks of Vitis vinifera. Asexual morph: Conidiophores were hyaline and penicillate. Conidia were hyaline, cylindrical, one septate or aseptate, with dimensions of 5.3-9.5 $\times 1.5-2.8 \mu \mathrm{m}$, mean $\pm \mathrm{SD}=7.8 \pm 1.1 \times 2.1 \pm 0.3 \mu \mathrm{m}$. The terminal vesicles were lageniform to ovoid. (Figure $4 \mathrm{c}$-d). Sexual morph: undetermined.

Culture characteristics: Colonies on PDA reached $74.9 \pm$ $0.8 \mathrm{~mm}$ diam. after $6 \mathrm{~d}$ incubation at $25^{\circ} \mathrm{C}$ in the dark, and were yellow to tan, with flourish aerial mycelium (Figure $4 \mathrm{a}-\mathrm{b}$ ).

Material examined: CHINA, Guangxi province, on trunk and rootstock of Vitis vinifera, 8 April 2018, Xinghong Li, living cultures, JZB3320001, JZB3320002.

Dactylonectria macrodidyma (Halleen, Schroers \& Crous) L. Lombard \& Crous

Pathogenic on trunks and roots of Vitis vinifera. Asexual morph: the isolates rarely formed chlamydospores and microconidia, producing abundant macroconidia on MEA. Macroconidia hyaline, cylindrical, straight to slightly curved, one to four septate, with dimensions 


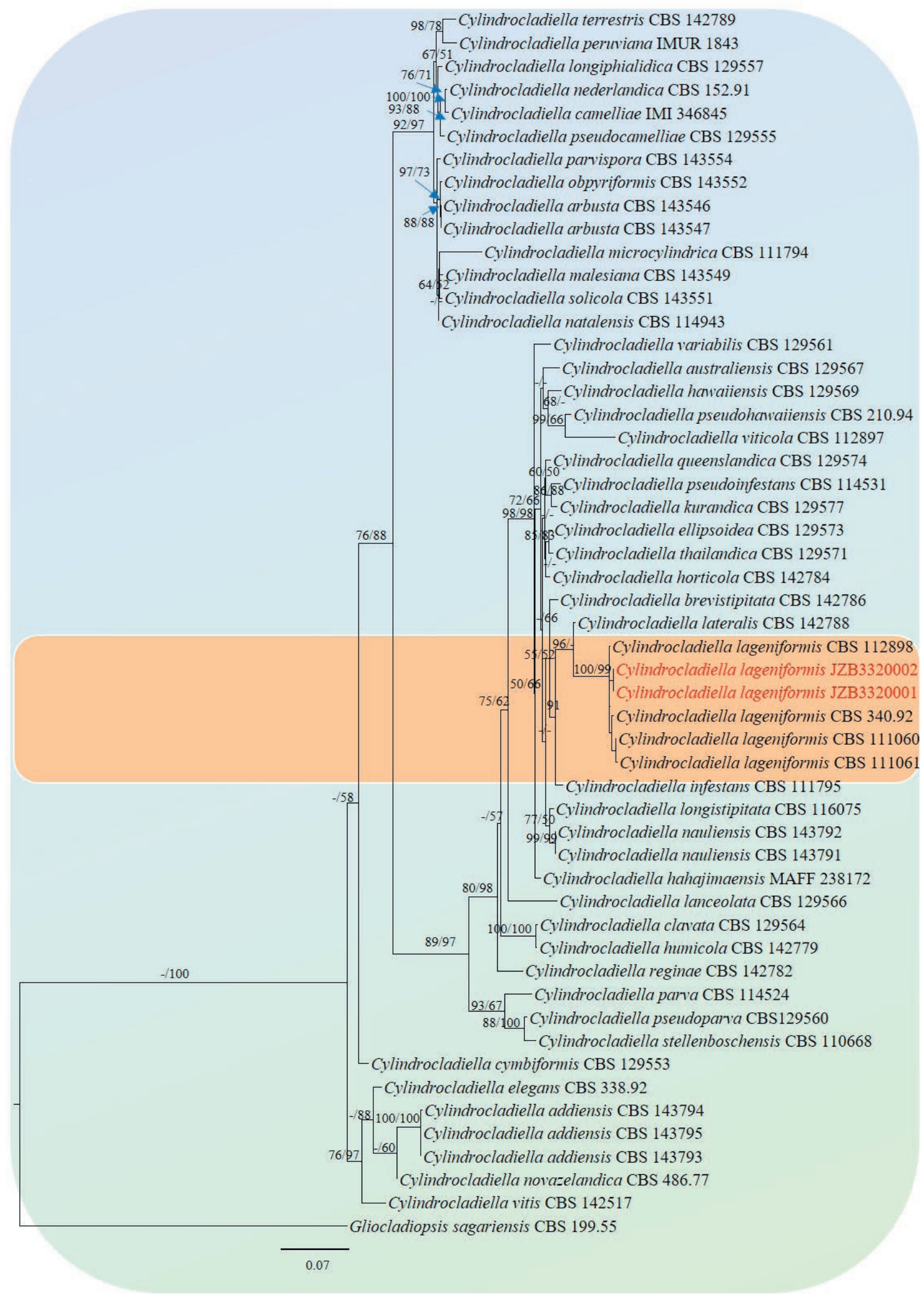

Figure 2. Maximum likelihood tree obtained from the phylogenetic analysis based on tef1, his 3 and tub2 sequence alignments. The scale bar represents 0.07 changes. The tree is rooted in Gliocladiopsis sagariensis (CBS 199.55). 


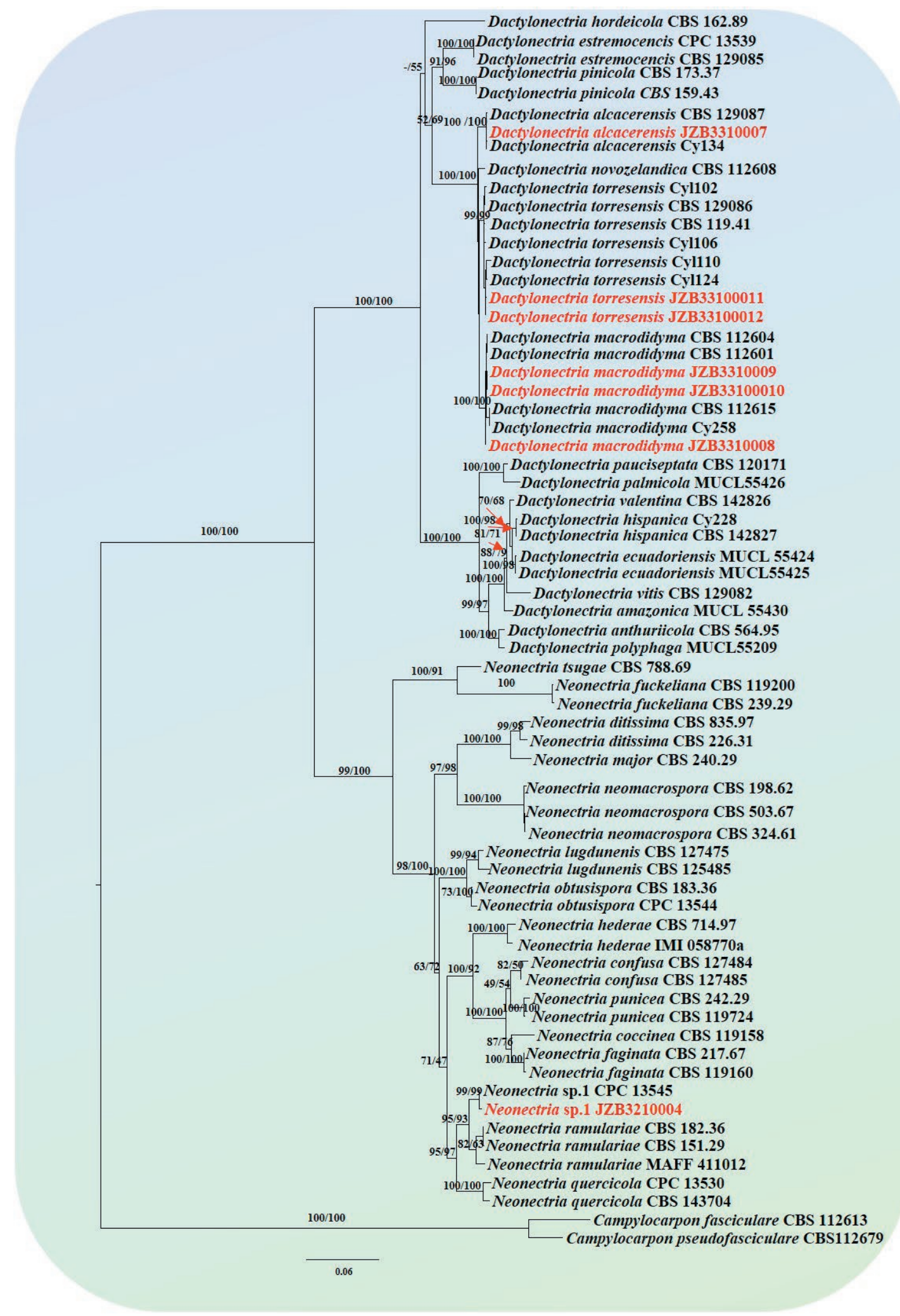

Figure 3. Maximum likelihood tree obtained from the phylogenetic analysis based on combined ITS, tub2, his3, and tef1 sequence alignments. The scale bar represents 0.05 changes. The ex-type strains are in bold font. The outgroups of the tree are Campylocarpon fasciculare (CBS 112613) and C. pseudofasciculare (CBS 112679). 

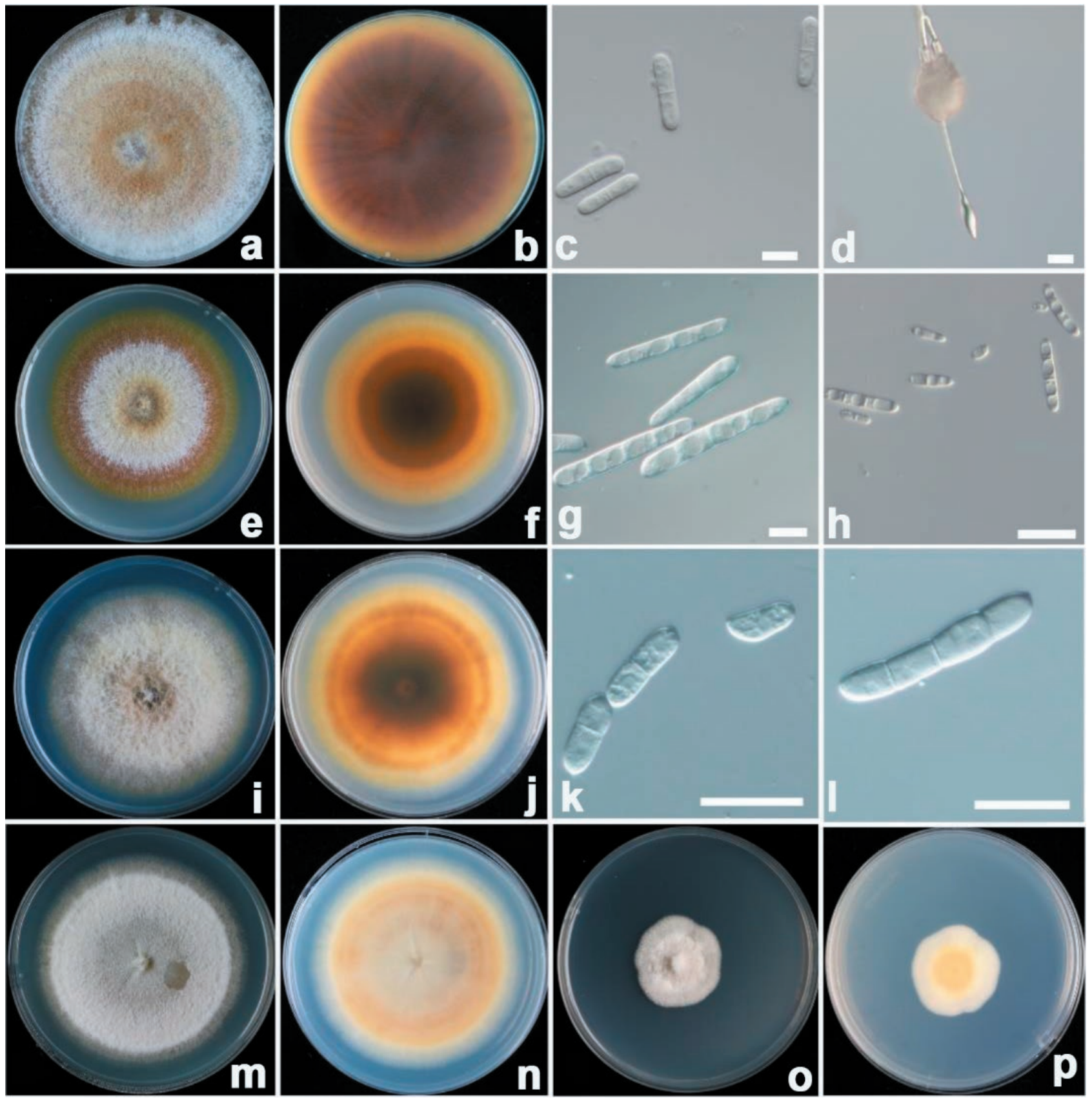

Figure 4. Photographs of isolated fungi and their morphological characterization. Colonies (top and bottom views of cultures) of Cylindrocladiella lageniformis (a and b), Dactinonectria macrodidyma (e and $\mathbf{f}), D$. torresensis (i and $\mathbf{j}), D$. alcaceresis $(\mathbf{m}$ and $\mathbf{n})$, or Neonectria sp.1 (o and $\mathbf{p}$ ). Conidia and terminal vesicles of C. lageniformis (c and $\mathbf{d}$ ). Conidia of D. macrodidyma ( $\mathbf{g}$ and $\mathbf{h}$ ), and D. torresensis (k and $\mathbf{l})$. All the fungi were grown on PDA for $14 \mathrm{~d}$. Bars $=10 \mu \mathrm{m}$ (c and d) or $20 \mu \mathrm{m}(\mathbf{g}, \mathbf{h}, \mathbf{k}$ and $\mathbf{l})$.

of $14.4-44.2 \times 4.0-8.2 \mu \mathrm{m}$, mean $\pm \mathrm{SD}=31.1 \pm 7.8 \times 6.2$ $\pm 0.9 \mu \mathrm{m}$ (Figure $4, \mathrm{~g}$ and $\mathrm{h}$ ). Microconidia ellipsoid to ovoid, hyaline, straight, aseptate to one septate. Sexual morph: undetermined.

Culture characteristics: Colonies on PDA reached $57.3 \pm$ $5.4 \mathrm{~mm}$ diameter after $9 \mathrm{~d}$ at $25^{\circ} \mathrm{C}$ in the dark, and were yellowish, with abundant aerial mycelium (Figure $4 \mathrm{e}$ ).
Colony reverse sides were burnt umber to raw sienna or brownish yellow on PDA (Figure 4f).

Material examined: CHINA, Ningxia province, on trunk and rootstock of Vitis vinifera, 8 April 2018, Qingtong Ye and Xinghong Li, living cultures, JZB3310008, JZB3310009, JZB3310010.

Dactylonectria torresensis (A. Cabral, Rego \& Crous) L. 
Lombard \& Crous

Pathogenic on trunks and rootstocks of Vitis vinifera. Asexual morph: The isolates rarely formed chlamydospores and microconidia, producing some macroconidia on MEA. Macroconidia straight or minutely curved, cylindrical, one to four septate. Microconidia zero to one septate, ellipsoid to ovoid. Sexual morph: undetermined.

Culture characteristics: Colonies on PDA reached 55.5 $\pm 3.6 \mathrm{~mm}$ diam. after $9 \mathrm{~d}$ at $25^{\circ} \mathrm{C}$ in the dark, and were pale buff to chestnut (Figure 4 , i and $\mathrm{j}$ ). Colony reverse sides were buff to umber to chestnut on PDA.

Material examined: CHINA, Shanxi and Hebei province, on trunk and rootstock of Vitis vinifera, 8 April 2018, Qingtong Ye and Xinghong Li, living cultures, JZB3310011, JZB3310012.

\section{Dactylonectria alcacerensis (A. Cabral, H. Oliveira \&} Crous) L. Lombard \& Crous

Pathogenic on roots of Vitis vinifera. Asexual morph: isolates did not produce macroconidia, microconidia, or chlamydospores on MEA. Sexual morph: undetermined. Culture characteristics: Colonies on PDA reached $49.3 \pm$ $2.2 \mathrm{~mm}$ diam. eafter $9 \mathrm{~d}$ at $25^{\circ} \mathrm{C}$ in the dark, and were felty to slightly cottony (Figure $4 \mathrm{~m}$-n).

Material examined: CHINA, Shanxi province, on trunk and rootstock of Vitis vinifera, 8 May 2018, Qingtong Ye and Xinghong Li, living culture, JZB3310007.

\section{Neonectria sp. 1}

Pathogenic on the bark of trunk of Vitis vinifera. Asexual morph: In the present study, the isolates did not produce macroconidia, microconidia, or chlamydospores on PDA. Sexual morph: undetermined.

Culture characteristics: Colonies on PDA reached $61.1 \pm 1.1 \mathrm{~mm}$ diameter after $15 \mathrm{~d}$ of incubation at $20^{\circ} \mathrm{C}$ in dark (Figure 4 o-p).

Material examined: CHINA, Xinjiang province, on trunk and rootstock of Vitis vinifera, 16 April 2018, Qingtong Ye and Xinghong Li, living culture,
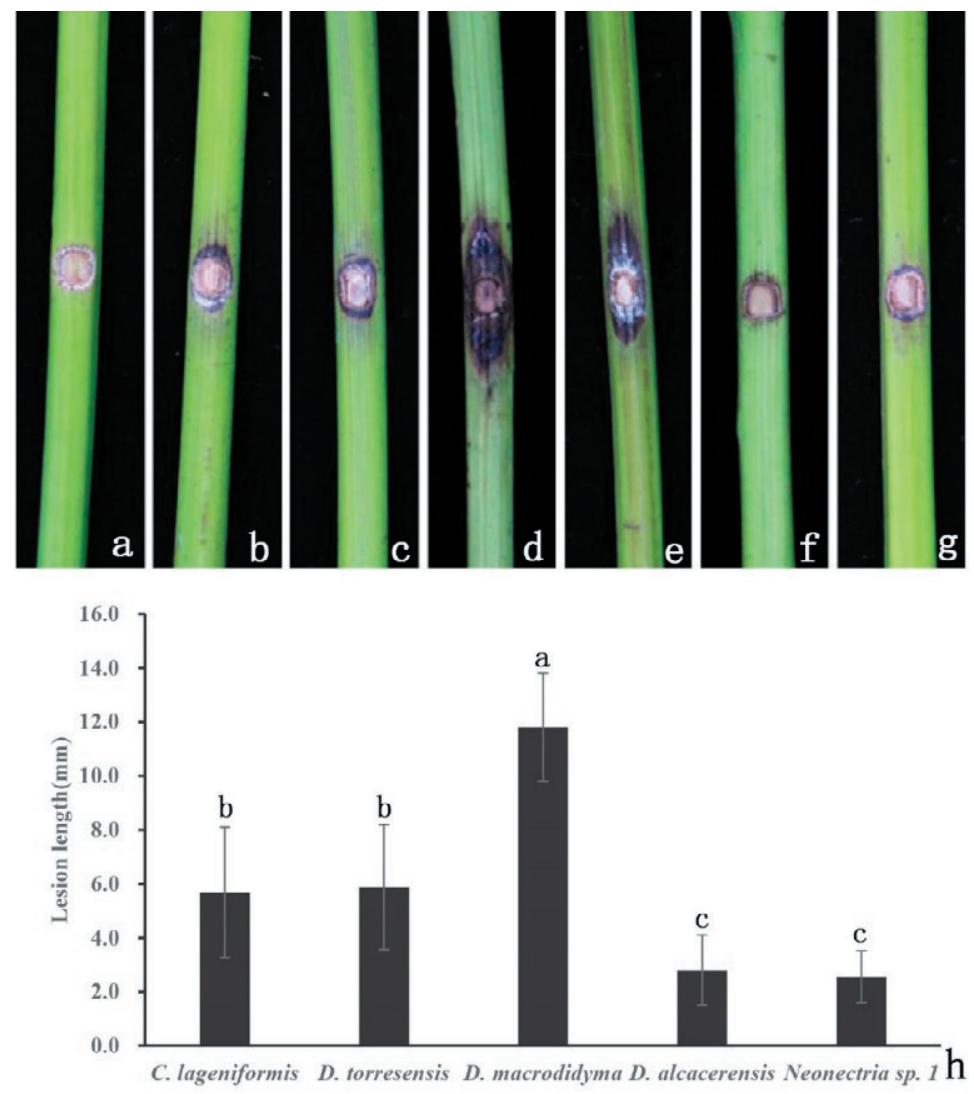

Figure 5. Pathogenicity tests results (after $7 \mathrm{~d}$ ) of BF fungal agents inoculated onto detached green shoots of grapevine 'Summer Black'. Control (a), Cylindrocladiella lageniformis(b), Dactinonectria torresensis (c), D. macrodidyma (d and e), D. alcacerensis(f), or Neonectria sp. $1(\mathbf{g})$. Histogram $(\mathbf{h})$ of mean lesion lengths on wood shoots after inoculations with the different fungi. Means accompanied by different letters are significantly different $(P=0.05)$. 
JZB3210004.

\section{Pathogenicity tests}

In the pathogenicity tests conducted with detached green shoots, the non-inoculated shoots did not develop any symptoms (Figure 5a). In contrast, shoots inoculated with mycelium discs resulted in necroses. The lesions were brown to black, and the mean lesion lengths differed among the different inoculated fungi $(P<0.05)$ (Figure 5, b to h). Dactylonectria macrodidyma was the most aggressive pathogen (mean lesion length $=1.18 \mathrm{~cm}$ ) among the five species (Figure 5). The re-isolation rates of isolates $C$. lageniformis, $D$. torresensis, $D$. macrodidy$m a, D$. alcacerensis, and Neonectria sp. 1 . were between $70 \%$ with $100 \%$ from the lesions.

Pathogenicity tests on 3-month-old grapevine cuttings showed different results for the different inoculated pathogens, as well. The non-inoculated controls showed no symptoms on the shoots (Figure 6a). Dacty- lonectria macrodidyma caused brown to black necrotic lesions on the shoots (mean lesion length $=1.95 \mathrm{~cm}$ ) (Figure 6, d and e). Less necrosis was observed in the cuttings inoculated in C. lageniformis, D. torresensis, Neonectria sp. 1., or D. alcacerensis (Figure 6, b to c', f to g'). The re-isolation rates of the different inoculated fungi from the respective lesions were between $70 \%$ with $100 \%$.

This is the first report of $C$. lageniformis, D. torresensis, D. macrodidyma, D. alcacerensis and Neonectria sp. 1 associated with BF of grapevines in China.

\section{DISCUSSION}

Grapevines can be affected by several diseases throughout each year, especially during fruit production. In the present study, 50 isolates obtained from diseased grapevine samples in five provinces of China were identified as C. lageniformis, D. torresensis, D. macrodidyma, D. alcacerensis, or Neonectria sp. 1. To date, D. torresen-
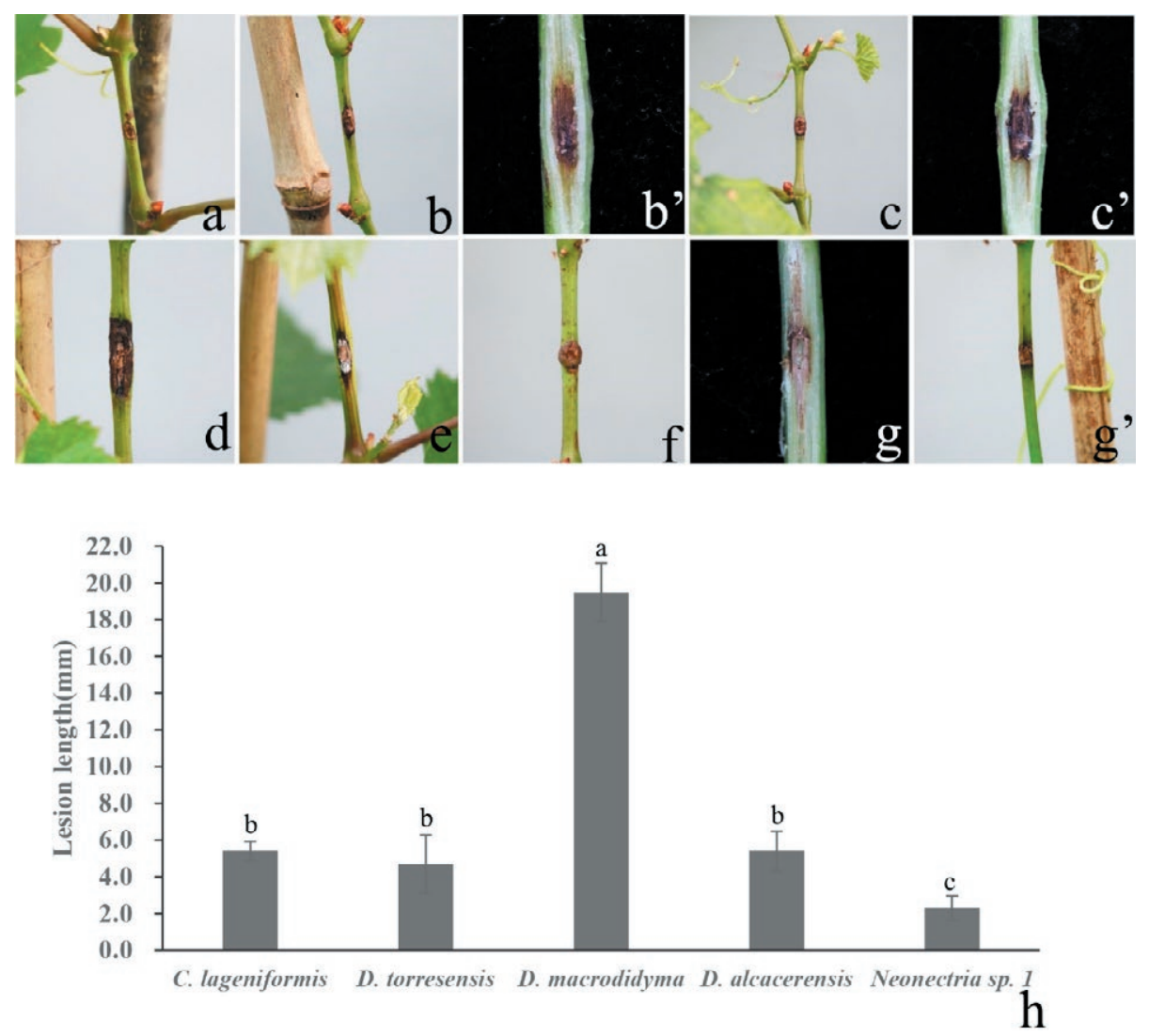

Figure 6. Pathogenicity tests results (after $80 \mathrm{~d}$ ) of BF fungal agents inoculated onto 3-month-old 'Summer Black' grapevine plants in greenhouse. Control (a), Cylindrocladiella lageniformis (b and b'), Dactinonectria torresensis(c and c'), D. macrodidyma (d and e), D. alcacerensis (f and $\mathbf{g})$, Neonectria sp. 1 ( $\left.\mathbf{g}^{\prime}\right)$. Histogram $(\mathbf{h})$ of mean lesion lengths caused by inoculations with the different fungi. Means accompanied by different letters are significantly different $(P=0.05)$. 
sis has been reported as grapevine pathogen in Australia, Canada, Czech Republic France, Italy, New Zealand, Portugal, South Africa, Spain and USA (Agustí-Brisach et al., 2013; Carlucci et al., 2017; Pecenka et al., 2018; Pintos et al., 2019), and Cylindrocladiella lageniformis has been reported mainly from California and South Africa (Van Coller et al., 2005; Koike et al., 2016).

The BF pathogens are soil-borne organisms that affect roots and the basal ends of rootstock vines, and most of the fungi reported in the present study were also reported in California and Spain (Koike et al., 2016; Berlanas et al., 2020). However, whole grapevines in the north grape-growing regions of China are routinely buried under the soil in winter, to allow survival during low temperature winter conditions. It has been proposed that the soil-borne pathogens could infect plants through wounds (to roots, rootstocks, trunks, canes and shoots). Pathogenicity tests of BF pathogens were carried out with canes or roots in previous studies (Koike et al., 2016; Berlanas et al., 2020). Nevertheless, pathogenicity tests of Cylindrocladiella lageniformis have also been conducted with green shoots (Van Coller et al., 2005; Koike et al., 2016). Therefore, in the present study, it was important to determine whether the soil-borne fungi could infect host canes or shoots. The fungi were inoculated by wounding between two nodes of each cutting. Among the tested fungi, $D$. macrodidyma produced the longest lesions in the pathogenicity tests. However, Berlanas et al. (2020) reported that virulence of D. alcacerensis was greater than that of D. macrodidyma and Neonectria sp. 1., while D. macrodidyma was found to be more virulent than $D$. alcacerensis in the present study. Differences in virulence of $D$. alcacerensis or the other species could be attributed to: (1) strain origins (Probst et al., 2019), (2) host genotype susceptibility to black foot fungus infections (Berlanas et al., 2020), (3) methods of inoculation (Alaniz et al., 2009b; Probst et al., 2019; Berlanas et al., 2020), or (4) inoculum dose.

The distribution of BF fungal pathogens in the present study may have been influenced by climate. The climate system of China is diverse due to the varied topography and vast area, including climates of Tibetan plateau, temperate continental, subtropical monsoon, and tropical monsoon (Yan et al., 2013). The characteristics of BF pathogens are likely to vary due to the diverse temperature of China. Based on the present study (data not shown), colony diameter on PDA after $6 \mathrm{~d}$ of $C$. lageniformis from the south of China reached up to $60.4 \pm 3.3 \mathrm{~mm}$ at $30^{\circ} \mathrm{C}$ while the other fungi ( $D$. torresensis, $D$. macrodidyma, $D$. alcacerensis and Neonectria sp. 1.) from the north of China hardly grew at $30^{\circ} \mathrm{C}$. Most of these fungi could grow below $5^{\circ} \mathrm{C}$ in PDA, except for C. lageniformis.
Although the incidence of diseased plants with BF symptoms was about $1 \%$ in the surveyed vineyards in China, which is much less than in France (losses of 50\%: Larignon et al., 1999), BF pathogens can infect grapevine roots and trunks in young nurseries and plantations, and the pathogenic fungi can be transmitted to new vineyards by cuttings (De la Fuente et al., 2016). The fungi $C$. lageniformis, $D$. torresensis, $D$. macrodidyma, $D$. alcacerensis, and Neonectria sp. 1. are all soilborne, and can infect hosts through the soil (Halleen et al., 2003). In the north of China, grapevines need to be buried under the soil for survival during cold weather, resulting in small wounds that are likely to be susceptible to infection by soilborne fungi, so more attention should be paid to BF in China in future.

Grapevine BF is prevalent in nurseries and new plantations (De la Fuente et al., 2016), and the current strategies for controlling this disease include good hygiene or sanitation, which are the most important means of obtaining healthy vines (Gramaje and Armengol 2011), including treatments with hot water, (Gramaje et al., 2010; Halleen and Fourie 2016), fungicides (Halleen et al., 2007; Rego et al., 2009; Alaniz et al., 2011) and biological control agents (Berbegal et al., 2020; Martínez-Diz et al., 2021; van Jaarsveld et al., 2020, 2021). Chemical treatments during propagation processes in nurseries for control of BF pathogens have been evaluated, including treating cutting prior to cold storage, cutting prior to callusing, rooting pre- and post-grafting, and pre-planting fungicide treatments of rooted cuttings, to eliminate or reduce potential fungal agents before planting (Halleen et al., 2007; Rego et al., 2009, Alaniz et al., 2011, Gramaje et al., 2018). Based on previous research, benomyl was effective for elimination or reducing Cylindrocarpon destructans infections (Rego et al. 2006). Reductions of $D$. torresensis and D. macrodidyma incidence and disease severity on the bases of 2 -year-old plants have been reported from applications of Streptomyces sp. E1+R4 before preplanting (MartínezDiz et al., 2021).

Some practices, such as hot water treatments, are useful for sanitizing commercially produced plants. Generally, this practice entails treating the plants at $50^{\circ} \mathrm{C}$ for $30 \mathrm{~min}$. However, this is stressful for the plants (Waite et al., 2013). Despite treated with these practices, diseases in symptomless plants can still be transmitted to non-infested areas (De la Fuente et al., 2016). The detection of BF fungi in soils or vines is essential for controlling the disease in nurseries and new plantations. Alaniz et al. (2009a) reported a multiplex PCR system for specific and early detection of Ilyonectria liriodendri (=Cylindrocarpon liriodendra), Dactylonectria macro- 
didyma (=Cylindrocarpon macrodidymum), and Dactylonectria pauciseptata (=Cylindrocarpon pauciseptatum) from pure fungus cultures or diseased plants. MartínezDiz et al. (2020) attempted to detect I. liriodendri in bulk soils, rhizosphere soils, and grapevine endorhizospheres using Droplet Digital PCR (ddPCR) and real-time PCR (qPCR) techniques. They showed that ddPCR was more sensitive than qPCR to lower target concentrations. Nevertheless, the ddPCR technique has not been used for detection of C. lageniformis, D. torresensis, D. macrodidyma, D. alcacerensis or Neonectria sp.1, and this technology could be useful for detection of BF in China. Further study should also be conducted to develop specific protocols for effective BF management.

\section{ACKNOWLEDGEMENTS}

The project was funded by 2018YFD0201301, CARS29 and JKZX201905. The authors thank the Institute of Forestry and Pomology, Beijing Academy of Agriculture and Forestry Sciences for providing 1-year-old 'Summer black' dormant shoots, and grapevine farmers for their co-operation.

\section{LITERATURE CITED}

Abreo E., Martínez S., Betucci L., Lupo S., 2010. Morphological and molecular characterization of Campylocarpon and Cylindrocarpon spp. associated with black foot disease of grapevines in Uruguay. Australasian Plant Pathology 39: 446-452.

Agustí-Brisach C., Armengol J., 2013. Black-foot disease of grapevine: an update on taxonomy, epidemiology and management strategies. Phytopathologia Mediterranea 52: 245-261.

Agustí-Brisach C., Gramaje D., García-Jiménez J., Armengol J., 2013. Detection of black-foot and Petri disease pathogens in natural soils of grapevine nurseries and vineyards using bait plants. Plant and Soil 364: 5-13.

Agustí-Brisach C., Mostert L., Armengol J., 2014. Detection and quantification of Ilyonectria spp. associated with black-foot disease of grapevine in nursery soils using multiplex nested PCR and quantitative PCR. Plant Pathology 63: 316-322.

Aigoun-Mouhous W., Elena G., Cabral A., León M., Sabaou N., ... Berraf-Tebbal A., 2019. Characterization and pathogenicity of Cylindrocarpon-like asexual morphs associated with black foot disease in Algerian grapevine nurseries, with the description of Pleio- carpon algeriense sp. nov. European Journal of Plant Pathology 154: 887-901.

Alaniz S., Leon M., García-Jiménez J., Abad P., Armengol, J., 2007. Characterization of Cylindrocarpon species associated with black-foot disease of grapevine in Spain. Plant Disease 91: 1187-1193.

Alaniz S., Armengol J., García-Jiménez J., Abad-Campos P., León M., 2009a. A multiplex pcr system for the specific detection of Cylindrocarpon liriodendri, $C$. macrodidymum, and C. pauciseptatum from grapevine. Plant Disease 93: 821-825.

Alaniz S., Armengol J., León M., Garcia-Gimenez J., Abad-Campos P., 2009b. Analysis of genetic and virulence diversity of Cylindrocarpon liriodendri and $C$. macrodidymum associated with black foot disease of grapevine. Mythological Research 113:16-23.

Alaniz S., Abad-Campos P., García-Jiménez J., Armengol J., 2011. Evaluation of fungicides to control Cylindrocarpon liriodendri and Cylindrocarpon macrodidymum in vitro, and their effect during the rooting phase in the grapevine propagation process. Crop Protection 30: 489-494.

Berbegal M., Ramón-Albalat A., León M., Armengol J., 2020. Evaluation of long-term protection from nursery to vineyard provided by trichoderma atroviride sc1 against fungal grapevine trunk pathogens. Pest Management Science 76: 967-977.

Berlanas C., Ojeda S., López-Manzanares B., AndrésSodupe M., Bujanda R., ... Gramaje D., 2020. Occurrence and diversity of black-foot disease fungi in symptomless grapevine nursery stock in Spain. Plant Disease 104: 94-104.

Carbone I., Kohn L. M., 1999. A method for designing primer sets for speciation studies in filamentous ascomycetes. Mycologia 91: 553-556.

Carlucci A., Lops F., Mostert L., Halleen F., Raimondo M. L., 2017. Occurrence fungi causing black foot on young grapevines and nursery rootstock plants in Italy. Phytopathologia Mediterranea 56: 10-39.

Crous P. W., Groenewald J. Z., Risede J. M., Simoneau P., Hywel-Jones N. L., 2004. Calonectria species and their Cylindrocladium anamorphs: species with sphaeropedunculate vesicles. Study in Mycology 50: 415-430.

Dissanayake A. J., Mei L., Wei Z., Zhen C., Udayanga D., ... Hyde K. D., 2015. Morphological and molecular characterisation of Diaporthe species associated with grapevine trunk disease in China. Fungal Biology 119: 283-294.

De la Fuente M., Fontaine F., Gramaje D., Armengol J., ... Corio-Costet M.F., 2016. Grapevine Trunk Diseases A Review. OIV Publications, 1st Edn. Paris. https:// 
www.oiv.int/public/medias/4650/trunk-diseasesoiv-2016.pdf

Glass N. L., Donaldson G., 1995. Development of primer sets designed for use with PCR to amplify conserved genes from Flamentous ascomycetes. Applied and Environmental Microbiology 61: 1323-1330.

Gramaje D., Alaniz S., Abad-Campos P., Garcia-Jimenez J., Armengol J., 2010. Effect of hot-water treatments in vitro on conidial germination and mycelial growth of grapevine trunk pathogens. Annals of Applied Biology 156: 231-241.

Gramaje D., Armengol J., 2011. Fungal trunk pathogens in the grapevine propagation process: potential inoculum sources, detection, identification, and management strategies. Plant Disease 95: 1040-1055.

Gramaje D., Úrbez-Torres J. R., Sosnowski M. R., 2018. Managing grapevine trunk diseases with respect to etiology and epidemiology: current strategies and future prospects. Plant Disease 102: 12-39.

Grasso S., Magnano Di San Lio G., 1975. Infezioni di Cylindrocarpon obtusisporum su piante di vite in Sicilia. Vitis 14: 38-39.

Guo L. D., Hyde K. D., Liew E. C. Y., 2000. Identification of endophytic fungi from Livistona chinensis based on morphology and rDNA sequences. New Phytologist 147: 617-630.

Hall T. A. 1999. BioEdit: a user-friendly biological sequence alignment editor and analysis program for Windows 95/98/NT. Nucleic Acids Symposium Series 41: 95-98.

Halleen F., Crous P. W., Petrini O., 2003. Fungi associated with healthy grapevine cuttings in nurseries, with special reference to pathogens involved in the decline of young vines. Australasian Plant Pathology 32: $47-52$.

Halleen F., Schroers H. J., Groenewald J. Z., Rego C., Oliveira H., Crous P. W., 2006. Neonectria liriodendri sp. nov. The main causal agent of black foot disease of grapevines. Studies in Mycology 55: 227-234.

Halleen F., Fourie P.H., Crous P. W., 2007. Control of black foot disease in grapevine nurseries. Plant Pathology 566: 37-645

Halleen F., Fourie P.H., 2016. An Integrated Strategy for the Proactive Management of Grapevine Trunk Disease Pathogen Infections in Grapevine Nurseries. South African Journal for Enology and Viticulture 37: 104-114.

Hofstetter V., Buyck B., Croll D., Viret O., Couloux A., Gindro K., 2012. What if esca disease of grapevine were not a fungal disease? Fungal Diversity 54: 51-67.

Katoh K., Rozewicki J., Yamada K. D., 2019. MAFFT online service: multiple sequence alignment, interac- tive sequence choice and visualization. Briefings in Bioinformatics 20: 1160-1166.

Kishino H., Hasegawa M., 1989. Evaluation of the maximum likelihood estimate of the evolutionary tree topologies from DNA sequence data, and the branching order in Hominoidea. Journal of Molecular Evolution 29: 170-179.

Koike S.T., Bettiga L.J., Nguyen T.T., and Gubler W.D., 2016. First report of Cylindrocladiella lageniformis and C. peruviana as grapevine pathogens in California. Plant Disease 100: 1783-1784.

Kuraku S., Zmasek C. M., Nishimura O., Katoh K. 2013. aLeaves facilitates on-demand exploration of metazoan gene family trees on MAFFT sequence alignment server with enhanced interactivity. Nucleic Acids Research 41: W22-W28.

Langenhoven S. D., Halleen F., Spies C. F. J., Stempien E., Mostert L., 2018. Detection and quantification of black foot and crown and root rot pathogens in grapevine nursery soils in the Western Cape of South Africa. Phytopathologia Mediterranea 57: 519-537.

Larignon P., 1999. Black foot disease in France. In: (Morton $\mathrm{L}$, ed.) Proceedings of the Seminar and Workshop on Black Goo Symptoms and Occurrence of Grape Declines. Fort Valley, VA, USA: International Ampelography Society 89-90.

Lawrence D. P., Nouri M. T., Trouillas F. P., 2019. Taxonomy and multi-locus phylogeny of Cylindrocarpon-like species associated with diseases roots of grapevine and other fruit and nut crops in California. Fungal Systematics and Evolution 4: 59-75.

Li H., Li R. Y., Wang H. 2007. New Disease for Winemaking Grape-Eutypa Dieback[J]. Liquor-Making Science \& Technology 155: 48-50.(in Chinese)

Li X. H., Yan J. Y., Kong F. F., Qiao G. H., Zhang Z. W., Wang Z. 2010. Botryosphaeria dothidea causing canker of grapevine newly reported in China. Plant Pathology 59: 1170-1170.

Lombard L., Van Der Merwe N. A., Groenewald J. Z., Crous P. W., 2014. Lineages in Nectriaceae: re-evaluating the generic status of Ilyonectria and allied genera. Phytopathologia Mediterranea 53: 515-532.

Manawasinghe I. S., Dissanayake A. J., Xing H.L., Mei L., Wanasinghe D. N., ... Ji Y.Y., 2019. High genetic diversity and species complexity of Diaporthe associated with grapevine dieback in China. Frontiers in Microbiology 10: 1936.

Marin-Felix Y., Hernández-Restrepo M., Wingfield M. J., Akulov A., Carnegie A. J., ... Crous P.W., 2019. Genera of phytopathogenic fungi: GOPHY 2. Studies in Mycology 92: 47-133. 
Martínez-Diz M. P., Andrés-Sodupe M., Berbegal M., Bujanda R., Gramaje D., 2020. Droplet digital PCR technology for detection of Ilyonectria liriodendri from grapevine environmental samples. Plant Disease 104: 1144-1150.

Martínez-Diz M.P., Díaz-Losada E., Andrés-Sodupe M., Bujanda R., Maldonado-González M.M., ... Gramaje D., 2021. Field evaluation of biocontrol agents against black-foot and Petri diseases of grapevine. Pest Management Science 77: 697-708.

O’Donnell K., Cigelnik E., 1997. Two divergent intragenomic rDNA ITS2 types within a monophyletic lineage of the fungus Fusarium are nonorthologous. Molecular Phylogenetics and Evolution 7: 103-116.

Pecenka J., Eichmeier A., Penazova E., Baranek M., Leon M., Armengol J. 2018. First report of Dactylonectria torresensis causing black-foot disease on grapevines in the Czech Republic. Plant Disease 102: 2038-2039.

Petit E., Barriault E., Baumgartner K., Wilcox W. F., Rolshausen P. E., 2011. Cylindrocarpon species associated with black-foot of grapevine in northeastern United States and Southeastern Canada. American Journal of Enology and Viticulture 62: 177-183.

Pintos C., Redondo V., Costas D., Aguin O., Mansilla P., 2019. Fungi associated with grapevine trunk diseases in nursery-produced Vitis vinifera plants. Phytopathologia Mediterranea 57: 407-424.

Probst C. M., Ridgway H. J., Jaspers M. V., Jones E. E., 2019. Pathogenicity of Ilyonectria liriodendri and Dactylonectria macrodidyma propagules in grapevines. European Journal of Plant Pathology 154: 405-421.

Rambaut A., FigTree v1.4.4: Tree figure drawing tool. 2018. https://github.com/rambaut/figtree/releases

Rego C., Oliveira H., Carvalho A., Phillips A., 2000. Involvement of Phaeoacremonium spp. and Cylindrocarpon destructans with grapevine decline in Portugal. Phytopathologia Mediterranea 39: 76-79.

Rego C., Farropas L., Nascimento T., Cabral A., Oliveira H. 2006. Black foot of grapevine, sensitivity of Cylindrocarpon destructans to fungicides. Phytopathologia Mediterranea 45: 93-100.

Rego C., Nascimento T., Cabral A., Silva M. J., Oliveira H., 2009. Control of grapevine wood fungi in commercial nurseries. Phytopathologia Mediterranea 48: $128-135$.

Silvestro D., Michalak I., 2016. RaxmlGUI: a graphical front-end for RAxML. Retrieved at 29 December 2016, from http://sourceforge. net/projects/raxmlgui/.

Swofford D. L., 2002. PAUP* 4.0: phylogenetic analysis using parsimony (and other methods). Sinauer Associates, Sunderland, Massachusetts, USA.
Udayanga, D., Liu, X. Z., Crous, P. W., McKenzie, E. H. C., Chukeatirote, E., and Hyde, K. D., 2012a. A multi-locus phylogenetic evaluation of Diaporthe (Phomopsis). Fungal Diversity 56: 157-171.

Udayanga D., Liu X. X., Crous P. W., McKenzie E. H. C., Chukeatirote E., Hyde K. D., 2012b. Multilocus phylogeny of Diaporthe reveals three new cryptic species from Thailand. Cryptogamie Mycologie 33: 295-309.

Van Coller G.J., Denman S., Groenewald J.Z., Lamprecht S.C., and Crous P.W., 2005. Characterisation and pathogenicity of Cylindrocladiella spp. associated with root and cutting rot symptoms of grapevines in nurseries. Australasian Plant Pathology 34: 489-498.

van Jaarsveld W. J., Halleen F., Mostert L., 2020. In vitro screening of Trichoderma isolates for biocontrol of black foot disease pathogens. Phytopathologia Mediterranea 59: 465-471.

van Jaarsveld, W. J. , Halleen F. , Bester M. C., Pierron R. J., Stempien E., Mostert L., 2021. Investigation of Trichoderma species colonization of nursery grapevines for improved management of black foot disease. Pest Management Science 77: 397-405.

Waite H., May P., Bossinger G., 2013. Variations in phytosanitary and other management practices in Australian grapevine nurseries. Phytopathologia Mediterranea 52: 369-379.

White T. J., Bruns T., Lee S., Taylor J., 1990. Amplification and direct sequencing of fungal ribosomal RNA genes for phylogenetics. In: PCR Protocols: A Guide to Methods and Applications. M.A. Innis, D.H. Gelfand, J.J. Sninsky and T.J. White, eds. Academic Press Inc., New York, NY, USA, 315-322.

Wilcox W. F., Gubler W. D., Uyemoto J. K., 2006. Compendium of Grape Diseases, Disorders, and Pests, Second Edition. American Phytopathological Society, St. Paul, Minnesota, USA, 232 pp.

Yan J. Y., Yue X., Wei Z., Yong W., ... Xing H.L., 2013. Species of Botryosphaeriaceae involved in grapevine dieback in China. Fungal Diversity 61: 221-236.

Ye Q.T., Manawasinghe I.S., Wei Z., Mugnai L., Hyde K.D., Xing H.L., Ji Y.Y., 2020. First Report of Phaeoacremonium minimum associated with grapevine trunk diseases in China. Plant Disease 104: 12591259. 